Rev Soc Esp Dolor

2015; 22(6): 282

\section{A propósito de las revisiones...}

\section{Sr. Director:}

Tiempo atrás publiqué en esta sección un artículo denominado ¿Revisión sistemática, revisión narrativa o metaanálisis? (1). El propósito del documento, estaba orientado a exponer en términos "sencillos" y de forma "escueta" la definición y utilidad de cada uno de los diseños mencionados anteriormente.

Si consideramos la gran cantidad de información que existe hoy en día (fenómeno de infoxicación), se hace necesario que los clínicos, estudiantes e investigadores desarrollen "ciertas" habilidades, tanto en la búsqueda y análisis crítico (2) de la literatura científica, así se podría asegurar la "supervivencia" entre tanta información de calidad dispar o no concluyente, convergiendo en un incremento de la incertidumbre clínica al momento que debemos decidir que opción terapéutica seguir.

En este campo, hoy en día juega un rol crucial las revisiones (diseños metodológicos que están orientados a resumir la información existente sobre un tema particular). Si estamos inmersos en el ámbito de la terapia, es sabido por todos que los Estudios clínicos aleatorizados son el patrón de oro para responder a este tipo de preguntas, el problema es el siguiente... al momento de buscar en bases de datos, es muy poco probable que encontremos sólo con un estudio clínico aleatorizado que responda a mi pregunta de interés. Para plasmar de mejor forma lo mencionado, cito el siguiente ejemplo: como clínico, me interesa saber si la Terapia Manual disminuye el dolor lumbar, por tal motivo, realizo una búsqueda específica en la base de datos MEDLINE, utilizando la siguiente combinación de términos MesH: ("Low Back Pain"[Mesh]) AND "Musculoskeletal Manipulations"[Mesh]; luego aplico el filtro metodológico "Clinical Queries", obteniendo los siguientes resultados: Estudios clínicos aleatorizados $=411$; Revisiones sistemáticas $=103$ (la búsqueda se realizó el 28 de junio, 2015). Al parecer, no sería tan razonable leer los estudios clínicos aleatorizados, considerando que son 411 (si tomamos en consideración el tiempo que tardaríamos en su lectura, análisis crítico, etc.). Si miramos la cantidad de revisiones sistemáticas de estudios clínicos, al parecer estaría ocurriendo lo mismo... ¿Qué hacemos en estos casos?, ¿serán todas las revisiones sistemáticas de buena calidad metodológica?, ¿todas concluirán lo mismo? Lo más probable, es que la respuesta sea "no". Es por esto, que la Colaboración Cochrane actualmente está impulsando un diseño metodológico denominado "overview o resúmenes de revisiones sistemáticas" (3).

Los overview o resúmenes de revisiones sistemáticas tienen como objetivo principal resumir múltiples revisiones sistemáticas (Cochrane y no Cochrane) de intervención y abordar el efecto de dos o más tratamientos potenciales sobre un problema de salud o afección, permitiendo un acceso más resumido de la evidencia científica existente sobre un problema particular (3).

Nuestro grupo de investigación tuvo el agrado de publicar su primer overview de revisiones sistemáticas de estudios clínicos aleatorizados en la presente revista (4), aprovechamos de agradecer al Director y a sus colaboradores, por aceptar este tipo de diseños metodológicos debido a que en un futuro no lejano, las decisiones clínicas (5) se podrán simplificar en gran medida con este tipo de diseño.

R. Aguilera-Eguía

Facultad de Ciencias de la Actividad Física. Universidad San Sebastián. Santiago, Chile

\section{Bibliografía}

1. Aguilera-Eguía RA. ¿Revisión sistemática, revisión narrativa o metaanálisis?. Rev Soc Esp Dolor 2014;21(6):359-60.

2. Aguilera-Eguía RA. Carta al Editor: Referente a la valoración crítica. Rev Ciencia e Innovación en Salud 2013;1(2):94.

3. Higgins JPT, Green S, editors. Cochrane Handbook for Systematic Reviews of Interventions Version 5.1.0 [updated March 2011]. The Cochrane Collaboration 2011.

4. Aguilera Eguía RA, Zafra Santos EO, Rojas López DK, Saavedra Rozas PA, Cofre Bolados C. Efectividad del láser de baja frecuencia en el tratamiento del dolor en pacientes con epicondilalgia lateral: un overview de revisiones sistemáticas. Rev Soc Esp Dolor 2015;22(1):39-47.

5. Aguilera-Eguía RA. ¿En qué basamos nuestras decisiones clínicas? Rev Soc Esp Dolor 2014;21(5):294. 